\title{
Fish Species Detection Application (FiSDA) in Leyte Gulf Using Convolutional Neural Network
}

\author{
Gil Gabornes Dialogo ${ }^{1,}$, Larmie Santos Feliscuzo ${ }^{2}$, Elmer Asilo Maravillas $^{2}$ \\ ${ }^{1}$ College of Computer Studies, Eastern Samar State University, Samar, Philippines \\ ${ }^{2}$ College of Computer Studies, Cebu Institute of Technology-University, Cebu, Philippines \\ Received 15 June 2021; received in revised form 26 July 2021; accepted 27 July 2021 \\ DOI: https://doi.org/10.46604/peti.2021.7892
}

\begin{abstract}
This study presents an application that employs a machine-learning algorithm to identify fish species found in Leyte Gulf. It aims to help students and marine scientists with their identification and data collection. The application supports 467 fish species in which 6,918 fish images are used for training, validating, and testing the generated model. The model is trained for a total of 4,000 epochs. Using convolutional neural network (CNN) algorithm, the best model during training is observed at epoch 3,661 with an accuracy rate of $96.49 \%$ and a loss value of 0.1359 . It obtains $82.81 \%$ with a loss value of 1.868 during validation and $80.58 \%$ precision during testing. The result shows that the model performs well in predicting Malatindok and Sapsap species, after obtaining the highest precision of $100 \%$. However, Hangit is sometimes misclassified by the model after attaining $55 \%$ accuracy rate from the testing results because of its feature similarity to other species.
\end{abstract}

Keywords: fish species, fish detection, mobile application, convolutional neural network

\section{Introduction}

The Leyte Gulf is among the major fishing grounds in the Philippines with a shelf area of about 2,724 square kilometers, covering the islands of Samar and Leyte. It is home to more than 467 different fish species [1]. Fishes are cold-blooded animals, typically with backbone, gills, fins, and lungs. They range from about 15,000 to 17,000 species [2]. Fishes have many kinds and have varied colors, shapes, and sizes [3]. Fish species recognition is a multi-class classification problem and is a compelling field of study that employs machine learning and computer vision [4]. Some researchers noticed that detection is a crucial part of a fish classification and counting system [5]. Moreover, manual species identification is not only time-consuming but also prone to misclassification especially in the Leyte Gulf, in which over 467 fish species exist [6].

There is relatively poor documentation for most groups of fishes, and the information on the inventory of species present within the gulf is especially scarce. Thus, employing computer vision and machine learning in fish species identification with developed technologies would transform marine science [7]. Various promising techniques for the identification of fishes emerged particularly in genetics, interactive computer software, image recognition, hydro-acoustics, and morphometry [8]. Recently, there is a paradigm shift of set-based classification for object recognition [9]. The convolutional operation is frequently used in computer vision, especially for noise reduction and edge detection [10].

An automated system for the identification and classification of fish species was created using a reduced version of AlexNet based on deep convolutional neural networks (CNNs). The results show that the modified AlexNet model has achieved the testing accuracy of $90.48 \%$ while the original AlexNet model achieved $86.65 \%$ over the untrained dataset [11]. In addition, a CNN system for aquarium family fish species identification achieved $85.59 \%$ testing accuracy [12].

* Corresponding author. E-mail address: dialogogil1992@gmail.com 
Optical fish detection network was applied to a system that is capable of parameterizing fish schools in underwater images. This was based on deep learning object detection architectures, and carried out the task of fish detection, localization, and species classification using visual data obtained by underwater cameras. Based on the experiments, it successfully detects $66.7 \%$ of the fish included, and further classifies $89.7 \%$ correctly [13]. Rekha et al. [14] used CNN with different architectures to extract and analyze the features in the detection and classification of various fish species to help and protect endangered species. The system exhibits an accuracy of $90 \%$ and $92 \%$ on the detection and classification respectively.

On the other hand, Fabic et al. [15] used blob counting and shape analysis for fish detection, counting, and species classification from underwater video sequences to identify the two most common fish species found in the Tubbathaha reef in the Sulu Sea, Philippines. Moreover, a two-step deep learning approach was used for the detection and classification of temperate fishes without pre-filtering. It employed the You Only Look Once (YOLO) object detection technique. In the second step, it adopted CNN with squeeze-and-excitation (SE) architecture for classifying each fish in the image without pre-filtering. The system achieved an accuracy of $99.27 \%$ using the pre-training model. Using the post-training model, it obtained $83.68 \%$ and $87.74 \%$ with and without image augmentation [16].

Given the above challenges in fisheries, the agreed unifying strategic objective for the classification and identification of fish is to develop information and communications technology (ICT) software or systems such as mobile applications, which have quickly become useful tools and are widely used today for their diversity and portability [17].

Thus, this study intends to develop a handy mobile application to identify fish species present in Leyte Gulf. The mobile users can capture an unknown fish image to the developed mobile application, in which the proposed model embedded in the application will then attempt to recognize the fish species. The application displays the recognition results on the application's graphical user interface. Aside from helping the non-professional fish enthusiasts, the produced information is essential in the decision-making processes of fisheries, marine conservation managers, and scientists, as well as in the documentation of species present within the gulf.

\section{Materials and Methods}

\subsection{Data preparation}

The available dataset is collected from the Bureau of Fisheries and Aquatic Resources (BFAR) Regional Office No. VIII. The dataset includes the list of species in Leyte Gulf and their local names. The corresponding images of the various species come from the BFAR publications and Fishbase [18]. There are 6,918 images used covering 467 fish species present in the areas along the Leyte Gulf as shown in Fig. 1. These images are clustered into 35 classes according to their local names. A ratio of 80-10-10 of the images is allocated, i.e., 5, 548 images for training, 685 images for validation and testing respectively as presented in Table 1.

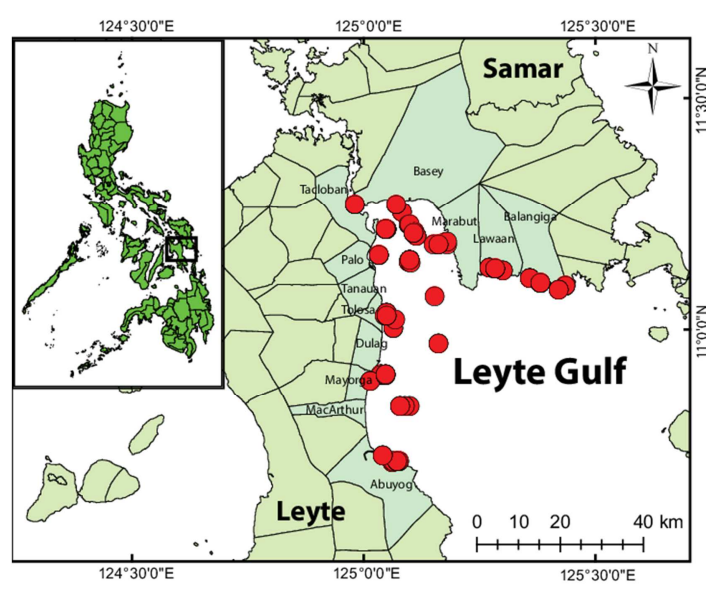

Fig. 1 Map of Leyte Gulf 
Table 1 Image allocation per class

\begin{tabular}{|c|c|c|c|c|}
\hline \multirow{2}{*}{ Class } & \multicolumn{3}{|c|}{ Number of images } & \multirow{2}{*}{ Total } \\
\cline { 2 - 4 } & Training & Validation & Testing & \\
\hline Abo & 109 & 13 & 13 & 135 \\
\hline Alho & 163 & 20 & 20 & 203 \\
\hline Alibangbang & 100 & 12 & 12 & 124 \\
\hline Baga-baga & 122 & 15 & 15 & 152 \\
\hline Bisugo & 87 & 11 & 11 & 109 \\
\hline Bon-ak & 184 & 24 & 24 & 232 \\
\hline Danggit & 316 & 40 & 40 & 396 \\
\hline Dumpilas & 199 & 8 & 8 & 215 \\
\hline Gangis & 102 & 13 & 13 & 128 \\
\hline Ganting & 120 & 15 & 15 & 150 \\
\hline Hamorok & 104 & 13 & 13 & 130 \\
\hline Hangit & 120 & 15 & 15 & 150 \\
\hline Katambak & 121 & 15 & 15 & 151 \\
\hline Kirawan & 140 & 17 & 17 & 174 \\
\hline Labungan & 179 & 23 & 23 & 225 \\
\hline Lapu-lapu & 320 & 40 & 40 & 400 \\
\hline Lubayan & 122 & 16 & 16 & 154 \\
\hline Malatindok & 102 & 13 & 13 & 128 \\
\hline Mamsa & 115 & 15 & 15 & 145 \\
\hline Mangagat & 119 & 15 & 15 & 149 \\
\hline Mol-mol & 305 & 38 & 38 & 381 \\
\hline Pakol & 162 & 21 & 21 & 204 \\
\hline Palad & 181 & 23 & 23 & 227 \\
\hline Panit & 100 & 13 & 13 & 126 \\
\hline Pating & 187 & 24 & 24 & 235 \\
\hline Sagisi-on & 88 & 11 & 11 & 110 \\
\hline Sapsap & 122 & 16 & 16 & 154 \\
\hline Siri & 182 & 23 & 23 & 228 \\
\hline Sulid & 118 & 15 & 15 & 148 \\
\hline Surahan & 239 & 30 & 30 & 299 \\
\hline Talakitok & 102 & 13 & 13 & 128 \\
\hline Tamban & 189 & 24 & 24 & 237 \\
\hline Tarukitok & 123 & 16 & 16 & 155 \\
\hline Ti-aw & 184 & 24 & 24 & 232 \\
\hline Tingag & 322 & 41 & 41 & 404 \\
\hline Grand total & 5,548 & 685 & 685 & 6,918 \\
\hline & & & & \\
\hline & & 13 \\
\hline
\end{tabular}

\subsection{Conceptual framework}

The application starts with the user capturing an image of fish using a mobile camera. The captured image will then be processed by the generated fish classification model. Finally, the application displays its prediction result with the details of the identified fish species as shown in Fig. 2.

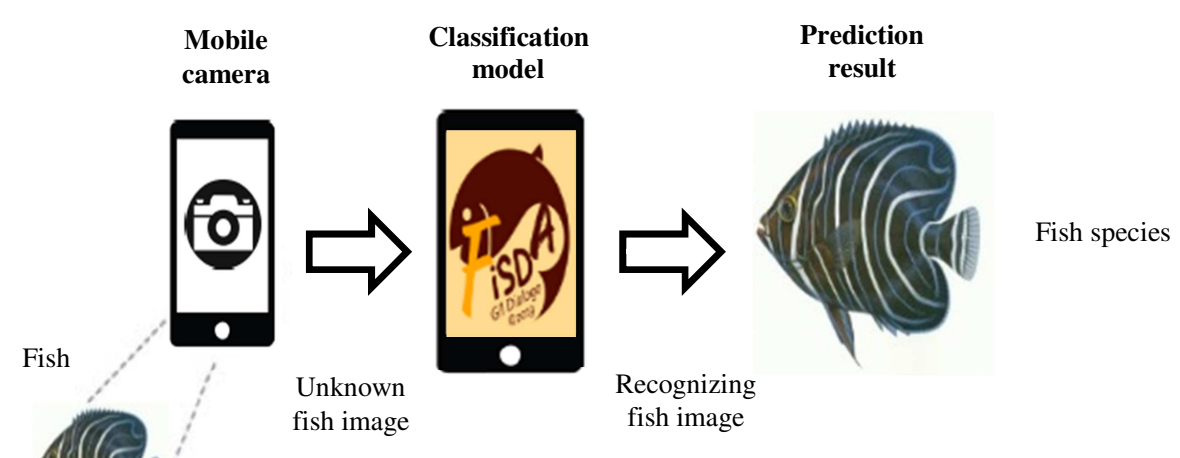

Fig. 2 Conceptual framework of the study 


\subsection{Building the CNN model}

The proposed model is built by using Python 3.6. The architecture of the deep network for the fish species identification is introduced in details in Fig. 3. It depicts the architecture for this study, which takes a fish image, processes the image, and then classifies the image under a certain type of fish species. The input image sequentially goes through a series of convolution-pooling layers for extracting low-level to high-level features and fully connected layers for mapping the extracted features into the final output.

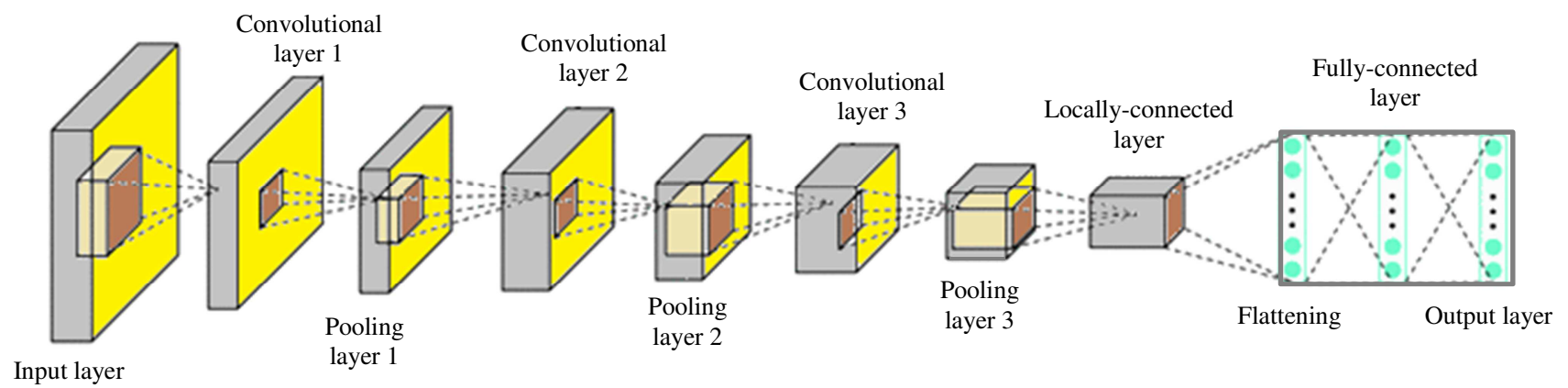

Fig. 3 CNN architecture

The convolutional layer contains learnable filters or kernels which are applied across the width and height of the input tensor. It then performs element-wise products between the entries of the filters and the input at any image positions and summed to obtain the feature maps. The output feature maps of convolution are passed through a rectified linear activation function, which returns the input directly if it is positive or zero as it receives any negative input. This function allows the model to learn faster and to perform better.

The pooling layer will then perform a downsampling operation, which progressively reduces the spatial size of the representation to decrease the number of subsequent learnable parameters as well as the computation in the network. In this study, max pooling with a filter of $2 \times 2$ and with a stride of 2 is applied, which outputs the maximum value in each patch extracted from the input feature maps.

The operations will be repeated until all the convolution-pooling layers have been finished, in which the final feature maps will be transformed to a one-dimensional array of numbers and connected to the fully connected or dense layers. The flattened output is being fed to a feed-forward neural network and applied backpropagation to every iteration of training. Over an iterated epoch, the model can distinguish between domination and certain low-level features in the images and classify them through the softmax activation function, wherein each value ranges between 0 and 1 , and all values sum up to 1 .

\subsection{Model performance evaluation}

The next phase is to determine how effective the model is, based on some basic performance metrics using the test dataset. The metrics include accuracy (Eq. (1)), precision (Eq. (2)), recall (Eq. (3)), and specificity (Eq. (4)).

$$
\begin{aligned}
& A=\frac{t p+t n}{t p+t n+f p+f n} \\
& P=\frac{t p}{t p+f p} \\
& R=\frac{t p}{t p+f n}
\end{aligned}
$$




$$
S=\frac{t n}{t n+f p}
$$

where $t p$ represents that when the actual class is true and the predicted is also true; $t n$ represents that when the actual class is false and the predicted is also false; $f p$ represents that when the actual class is false and the predicted is true; $f n$ represents that when the actual class is true and the predicted is false.

\section{Results and Discussion}

\subsection{CNN model for fish recognizer}

Fig. 4 illustrates that the $64 \times 64$ input image becomes $62 \times 62$ after the $3 \times 3$ filter in the first convolutional layer. It gets reduced in half after each pooling layer, from $31 \times 31$ on the first, then to $14 \times 14$ on the second, and $6 \times 6$ on the last pooling layer. This will then be flattened, resulting in 4,608 $(6 \times 6 \times 128)$, the shape of the data once it comes out of the convolutions. The first dense layer with 256 neurons has a total of 1,179,904 $(256 \times(4,608+1))$ parameters, while the second dense layer with 35 neurons as well has 8,995 parameters $(35 \times(256+1))$.

\begin{tabular}{|c|c|c|}
\hline Layer (type) & Output Shape & Param \# \\
\hline 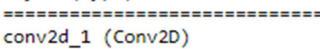 & $\begin{array}{l}=================== \\
\text { (None, } 62,62,32)\end{array}$ & $\begin{array}{l}======== \pm \\
896\end{array}$ \\
\hline activation_1 (Activation) & (None, 62, 62, 32) & $\theta$ \\
\hline max_pooling2d_1 (MaxPooling2 & (None, 31, 31, 32) & $\theta$ \\
\hline Conv2d_2 (Conv2D) & (None, 29, 29, 64) & 18496 \\
\hline activation_2 (Activation) & (None, 29, 29, 64) & $\theta$ \\
\hline max_pooling2d_2 (MaxPooling2 & (None, $14,14,64)$ & $\theta$ \\
\hline conv2d_3 (Conv2D) & (None, 12, 12, 128) & 73856 \\
\hline activation_3 (Activation) & (None, 12, 12, 128) & $\theta$ \\
\hline max_pooling2d_3 (MaxPooling2 & (None, $6,6,128$ ) & $\theta$ \\
\hline flatten_1 (Flatten) & (None, 4608) & $\theta$ \\
\hline dense_1 (Dense) & (None, 256) & 1179904 \\
\hline activation_4 (Activation) & (None, 256) & $\theta$ \\
\hline dense_2 (Dense) & (None, 35) & 8995 \\
\hline activation_5 (Activation) & (None, 35) & $\theta$ \\
\hline \multicolumn{3}{|l|}{$\begin{array}{l}====================== \\
\text { Total params: } 1,282,147 \\
\text { Trainable params: } 1,282,147 \\
\text { Non-trainable params: } \theta\end{array}$} \\
\hline
\end{tabular}

Fig. 4 Summary of model layers

A sequential neural network with input shape $(64,64$, and 3$)$ is configured wherein $64 \times 64$ represents the image dimension, while 3 indicates that the input image is colored (RGB). The network is composed of a linear stack of 3 sets of convolutional (Conv2D) - pooling (MaxPooling2D) layers before the dense or fully connected layers at the bottom. The Conv2D layers have 32, 64 , and 128 output channels respectively and a kernel size of $3 \times 3$. The activation function for each Conv2D layer is the Rectified Linear Unit (ReLU), followed by a MaxPooling2D layer, which reduces the number of parameters in the model by sliding a $2 \times 2$ pooling filter across the previous layer and taking the max values in the filter. Between the convolutional layers and the dense layers, there is a flatten layer that connects them. The first two dense layers both have 256 nodes, each activated by a ReLU function. The last dense layer has 35 nodes activated by the softmax activation function, which allows the output to be interpreted as probabilities. Thus, the model will take the class option, which obtains the highest probability.

Figs. 5-7 demonstrate the visualization of every channel for each intermediate activation phase. It shows how CNN finds the patterns in the images and how it carries the information from one layer to another layer. It can be noticed that the activations in the above layers retain almost all of the information present in the initial image. However, when the layers get more in-depth, the 
activations become increasingly abstract and less visually interpretable. The network begins to encode higher-level presentations which carry gradually less information about the visual contents of the image and more information related to the class of the image. There are instances that the filters are left blank, which indicates that they are not activated at all.

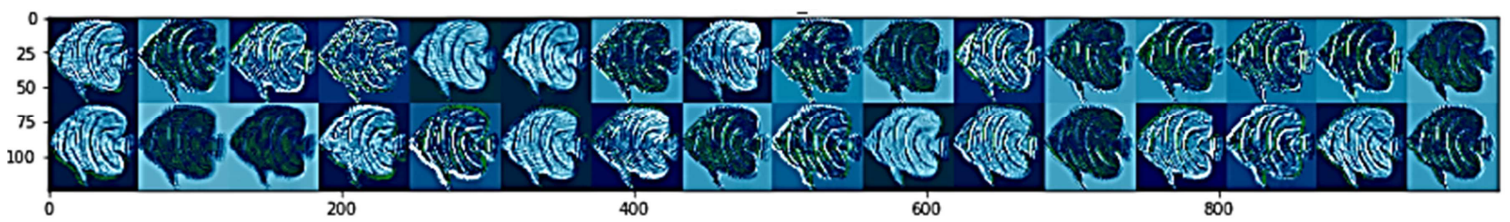

(a) Feature map from the first set of Conv2D layer

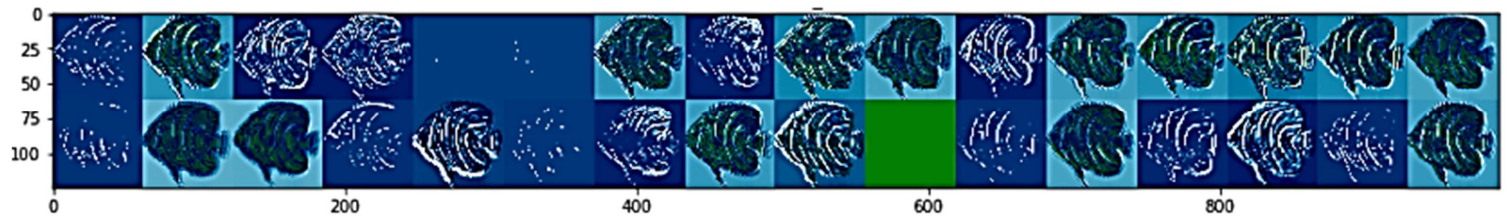

(b) Feature map from the first set of ReLU

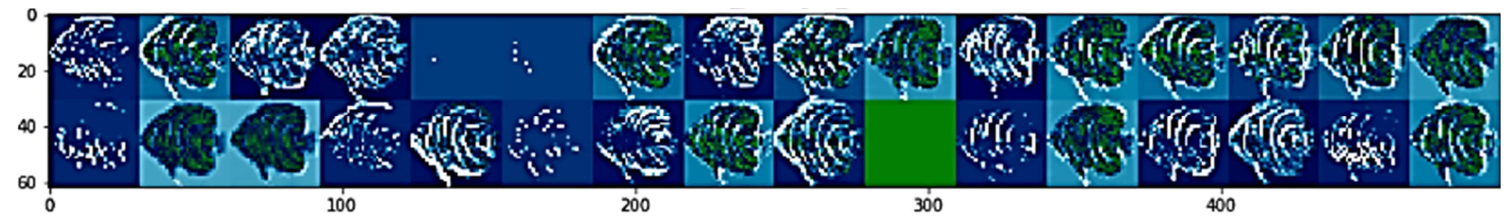

(c) Feature map from the first set of MaxPooling2D layer

Fig. 5 Visualization of feature maps from the first set of Conv + ReLU + Pool

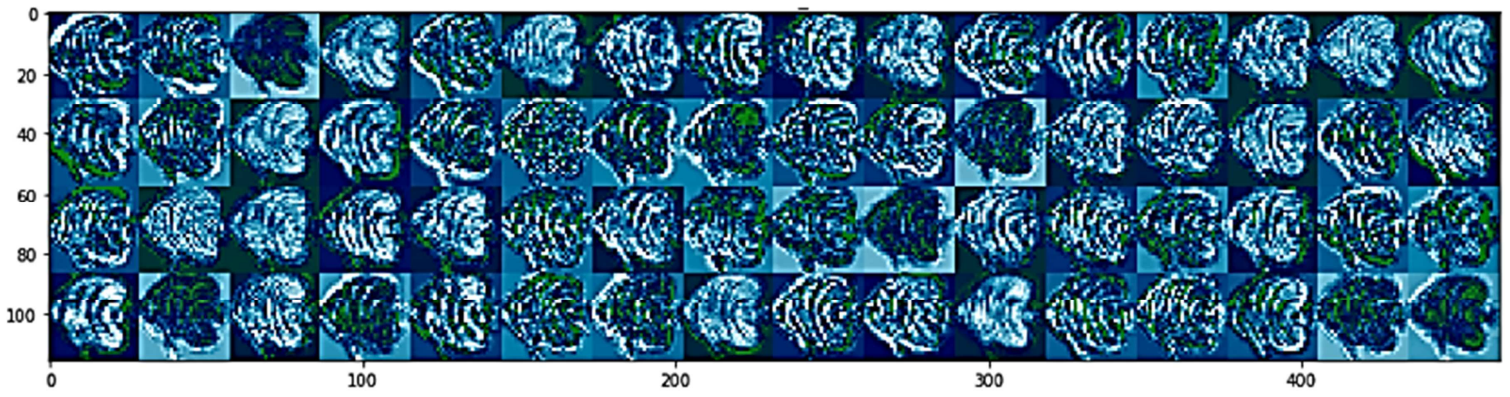

(a) Feature map from the second set of Conv2D layer



(b) Feature map from the second set of ReLU

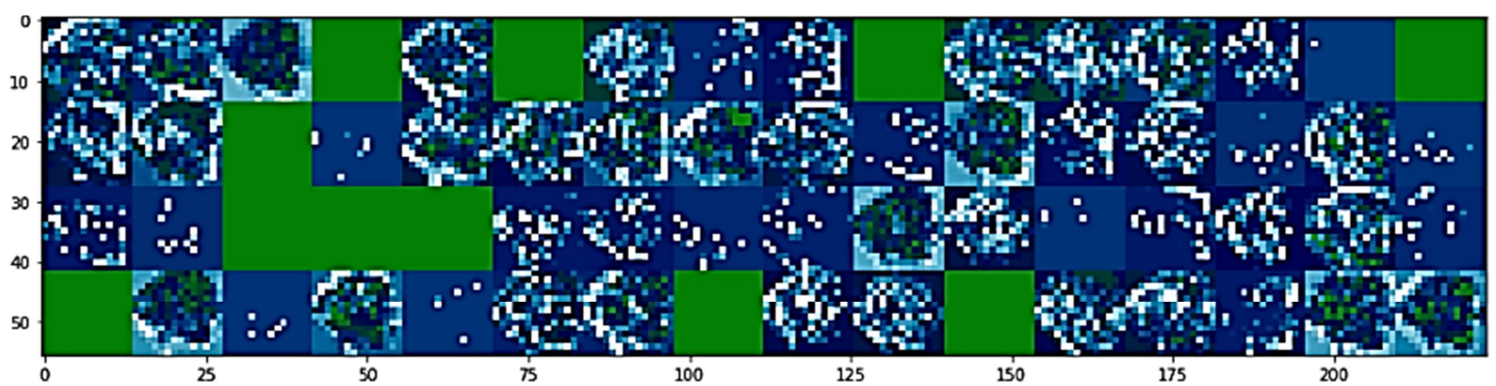

(c) Feature map from the second set of MaxPooling2D layer

Fig. 6 Visualization of feature maps from the second set of Conv + ReLU + Pool 


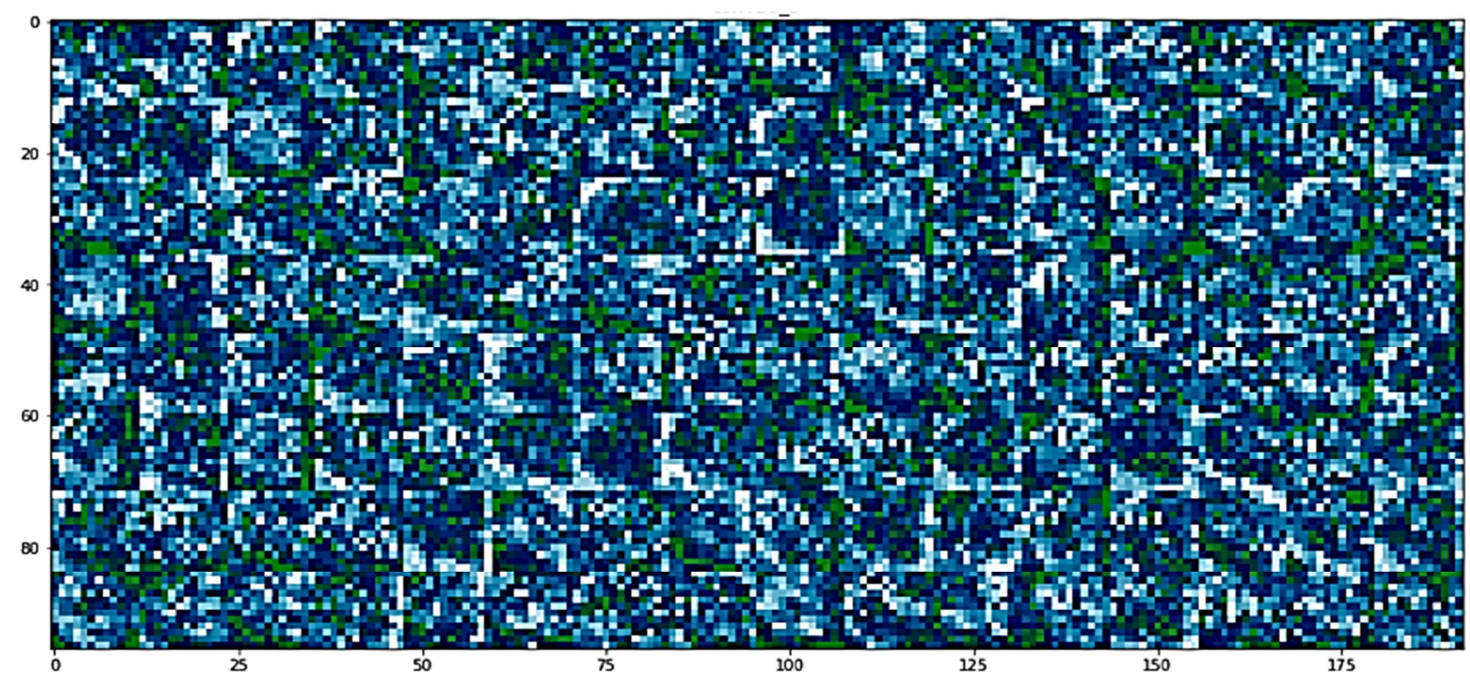

(a) Feature map from the third set of Conv2D layer

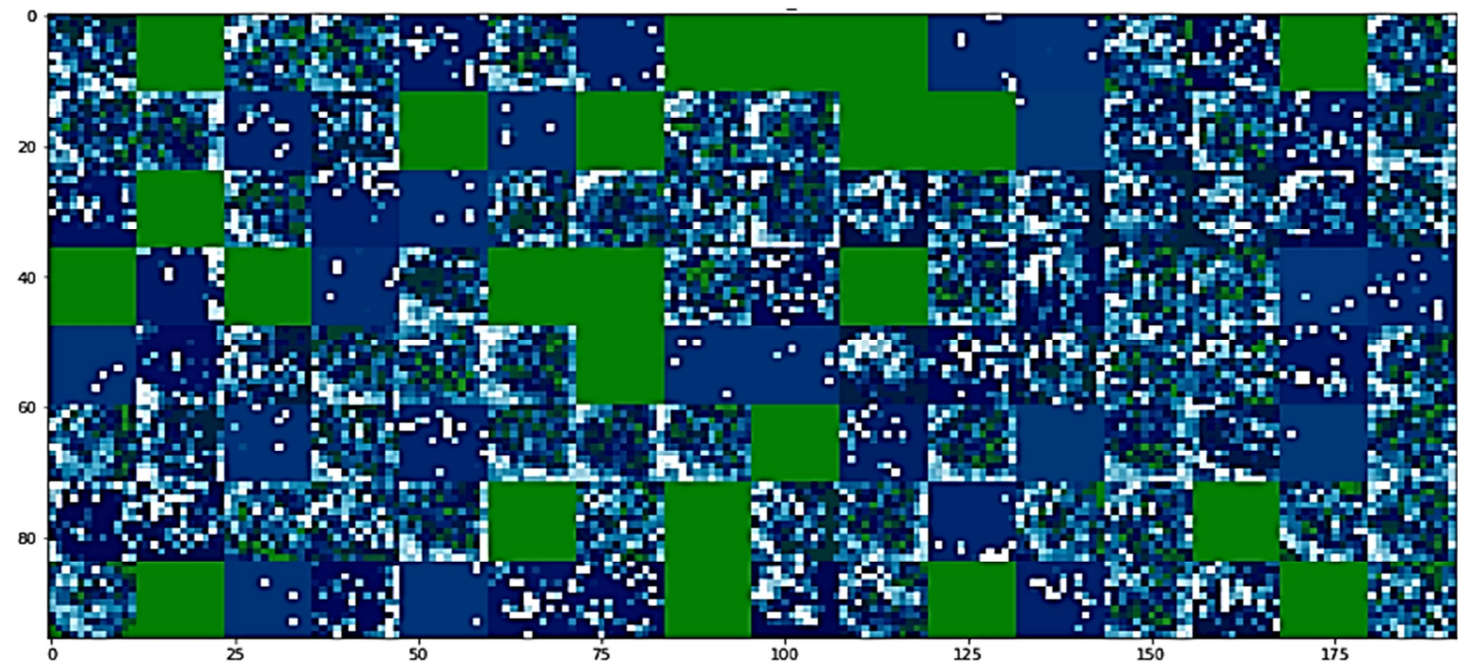

(b) Feature map from the third set of ReLU

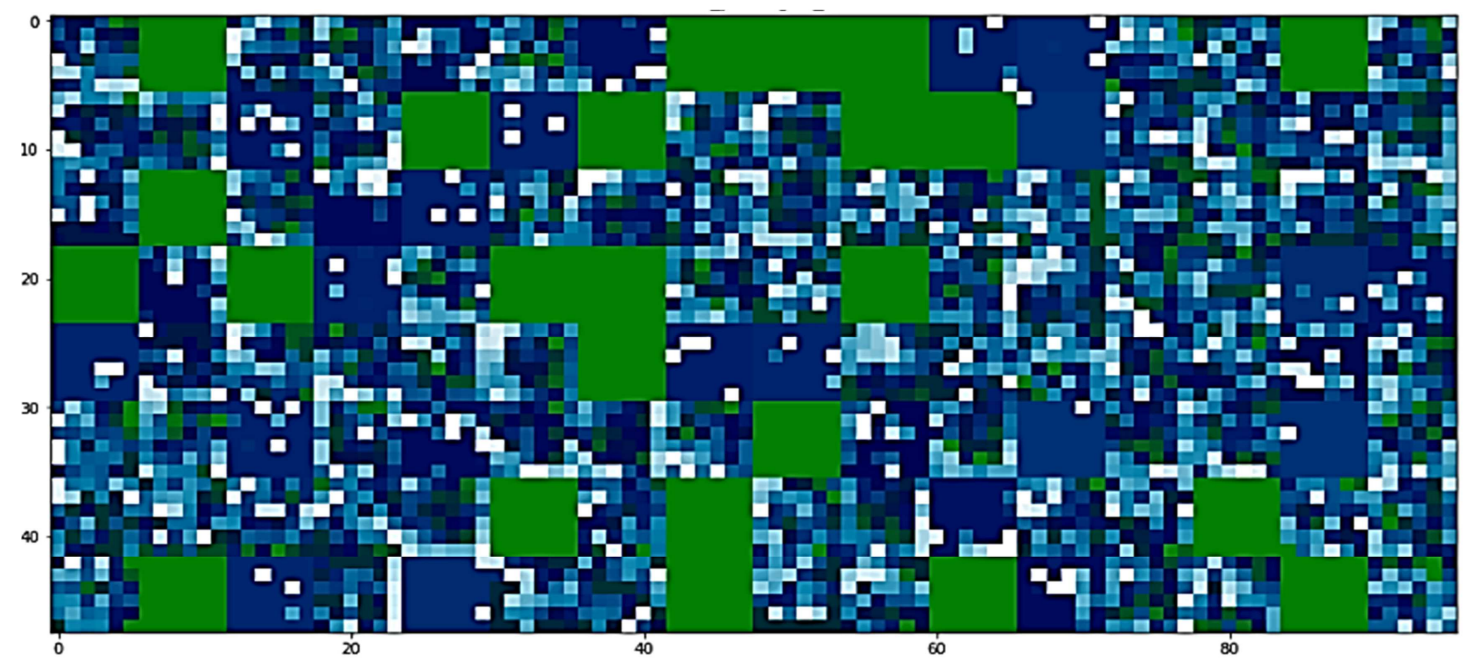

(c) Feature map from the third set of MaxPooling2D layer

Fig. 7 Visualization of feature maps from the third set of Conv + ReLU + Pool

Fig. 8 shows the training and validation accuracy as a function of the epoch. The accuracy metric is calculated to measure the algorithm's performance in an interpretable way. It is the measure of how accurate the model's prediction is compared to the actual data. 


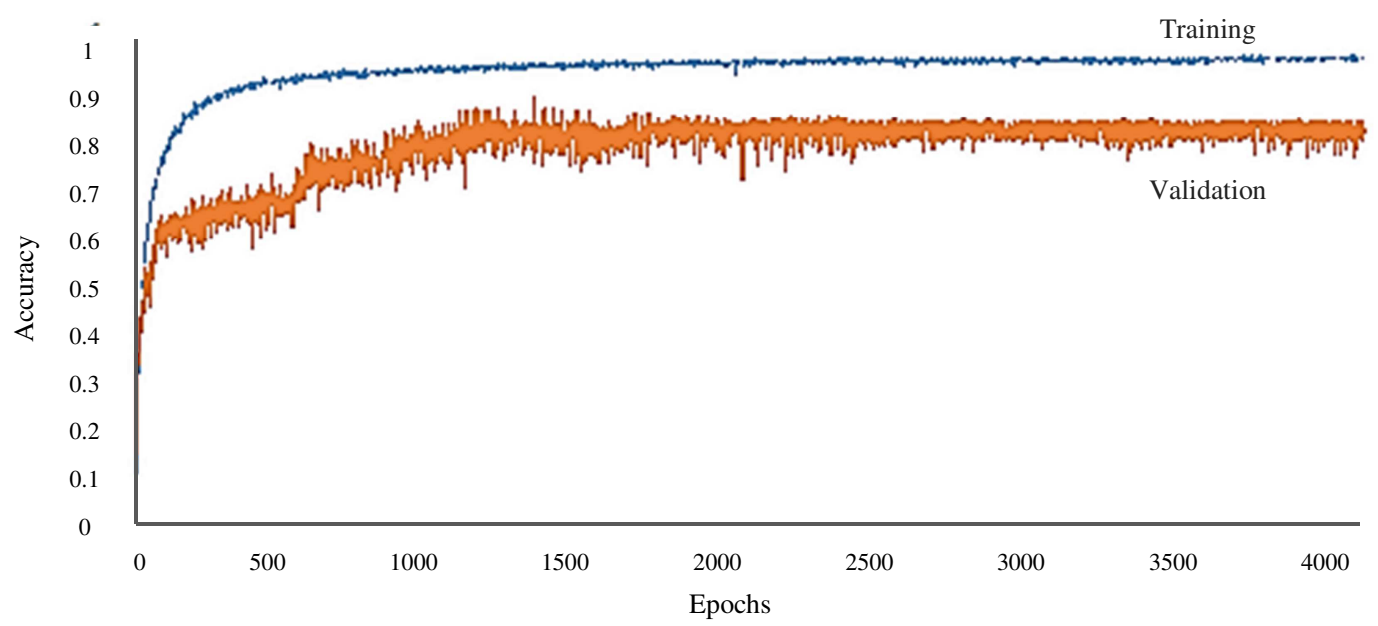

Fig. 8 Training accuracy plot of the CNN model

The loss is also calculated to serve as a reference in determining how well the model is during the training and validation phase. Unlike accuracy, a loss is not a percentage. It is the sum of errors made for each sample in training or validation sets, and implies how poorly or well a model behaves after each iteration of optimization. The loss function of the model is illustrated in Fig. 9, which shows that the loss for the training phase is nearly zero indicating that the model's prediction on the trained datasets is almost perfect. On the other hand, as to the validation phase, there is an occurrence of overfitting showing that the model does not affirmatively well in predicting new datasets.

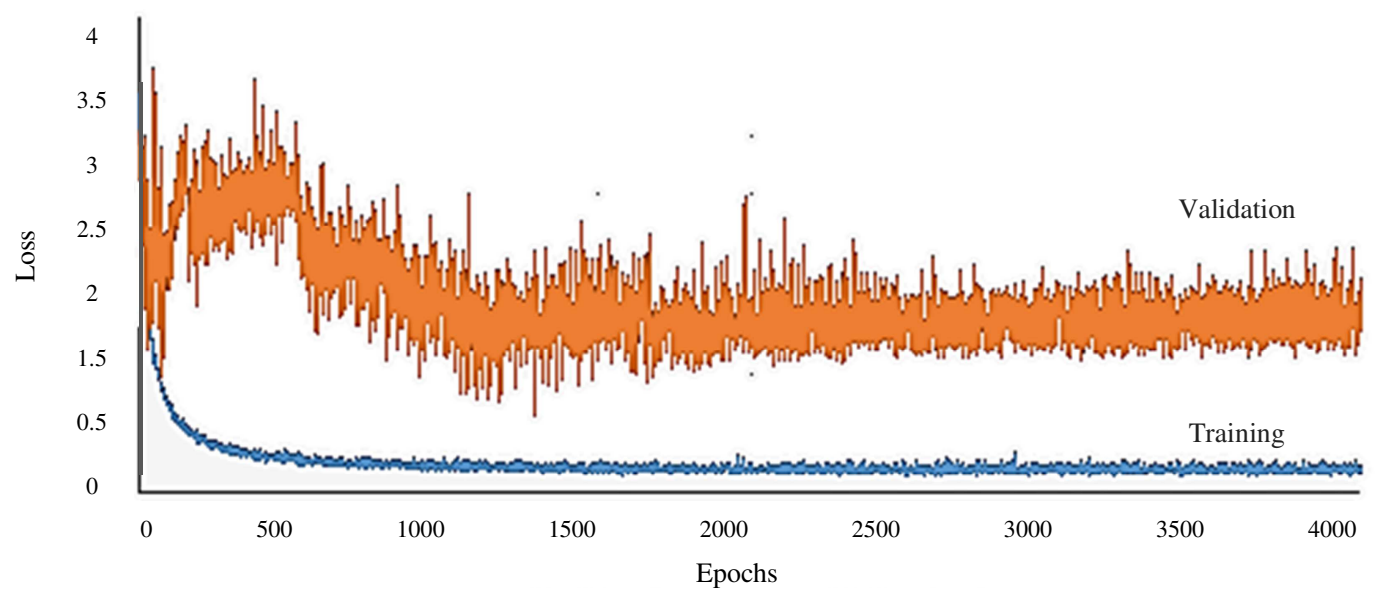

Fig. 9 Training loss plot of the CNN model

The model is trained for a total of 4,000 epochs. The best model during training is observed at epoch 3,661 obtaining an accuracy rate of $96.49 \%$ with a loss value of 0.1359 . However, the performance of the model for the validation set is only $82.81 \%$ with a loss value of 1.868 . The result implies that the model performs less well on the unseen dataset.

\subsection{Model performance}

The confusion matrix shown in Table 2 describes the performance of the model on the testing dataset. It displays the number of correct predictions (diagonal) made by the model, as well as the number of incorrect predictions (off-diagonal). Based on the generated confusion matrix, the model garners an accuracy of $80.58 \%$ during testing. As observed also, Malatindok and Sapsap are the best species predicted by the model since they obtain $100 \%$ accuracy. It is because of their distinct morphological features susch as shape and color. On the other hand, Hangit gains the least accuracy after it results in a 55\% precision rate. It means that Hangit is the most misclassified species by the model because of its feature similarity to other species. 
Table 2 Confusion matrix

\begin{tabular}{|c|c|c|c|c|c|c|c|c|c|c|c|c|c|c|c|c|c|c|c|c|c|c|c|c|c|c|c|c|c|c|c|c|c|c|c|c|}
\hline - & $\stackrel{8}{<}$ & 妾 &  & 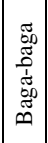 &  & 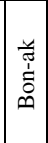 & 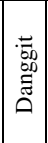 &  & 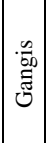 & 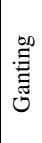 & 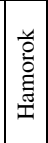 &  & 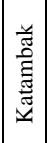 & 焉 & 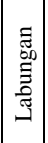 &  & 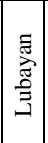 & 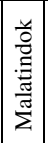 & 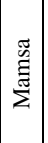 & 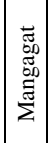 & $\begin{array}{l}\overline{0} \\
\bar{g} \\
\frac{1}{0} \\
\dot{0}\end{array}$ & 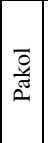 & $\mid \begin{array}{l}\vec{z} \\
\frac{\tilde{J}}{\tilde{\sigma}} \\
\tilde{\sigma}\end{array}$ & $\begin{array}{l}\text { 节 } \\
\text { 巳 }\end{array}$ & 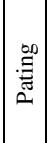 & 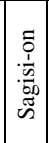 & 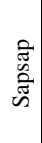 & $: \Xi$ & 咅 &  & 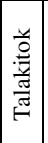 & 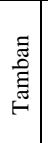 & 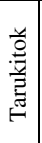 & 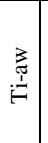 & 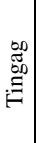 & \\
\hline Abo & $\simeq$ & & & & & & & & & & & & & & & 1 & & & & & & & & & & & & & & & & & & & & 9 \\
\hline Alho & 1 & $\underline{0}$ & & & & & & & & & 1 & & & & & & & & & & & 1 & & & & & & & & & & & 1 & & & ¿े \\
\hline Alibangbang & & & 인 & & & & & & & & & & & & & 1 & & & & & & & & & & & & & & & & & & & 1 & $\simeq$ \\
\hline Baga-baga & & & & 2 & & & & & & & & & & & 1 & & & & & & & & & & & & & & & & & & & & 1 & 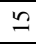 \\
\hline Bisugo & & & & & $\infty$ & 1 & & & & & & & & & & & & & & & & & & & & 1 & & & & & & & & 1 & & $=$ \\
\hline Bon-ak & & & & & & ते & 1 & & & & & & & & 1 & 1 & & & & & & & & & & & & 1 & & & & & & & & 4 \\
\hline Danggit & & & & & & & m & & & & & & & & & 1 & & & & & & 1 & & & & & & & & 2 & & & & & & q \\
\hline Dumpilas & & & & & & & & 0 & & & & & & & & & & & & & & 1 & & & & & & 1 & & & & & & & & $\infty$ \\
\hline Gangis & & & & & & & & & $=$ & & 1 & & & & & & & & & & & & & & & & & & & 1 & & & & & & 9 \\
\hline Ganting & & & & 2 & & & & & & 0 & & & & & 1 & 2 & & & & & & & & & & & & & & & & & & & & 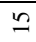 \\
\hline Hamorok & & & & & & & & & & \begin{tabular}{|l|}
1 \\
\end{tabular} & 우 & & & & & & & & & & & & & & & & & & & & & 1 & & & 1 & 9 \\
\hline Hangit & & & & & & & & & & & 1 & $=$ & & & & & & & & & & & & & 1 & & & & & & & & & & 2 & $\because$ \\
\hline Katambak & & & & & & 1 & & & & & & & $\simeq$ & 1 & & & & & & & & & & & & & & 1 & & & & & & & & 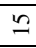 \\
\hline Kirawan & & & & 1 & & & 1 & 1 & & & 1 & & & $=$ & & 1 & & & & 1 & & & & & & & & & & & & & & & & $\Xi$ \\
\hline Labungan & & & & & & & 1 & 1 & & & & 1 & & & $\stackrel{\infty}{-}$ & & & & & 1 & & & & & & & & 1 & & & & & & & & $\pi$ \\
\hline Lapu-lapu & & & & & & & 2 & & & & & 2 & & & & $\bar{m}$ & & & & & & & 1 & & & & & & & & & & & & 4 & q \\
\hline Lubayan & & & 1 & & & & & & 1 & & & & & 1 & & & $\simeq$ & & & 1 & & & & & & & & & & & & & & & & $\underline{0}$ \\
\hline Malatindok & & & & & & & & & & & & 1 & & & & & & $a$ & 1 & & & & & & & & & & & & & 1 & & 1 & & 9 \\
\hline Mamsa & & & & & & & & & & & & & & & & & & & 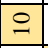 & & & 1 & & & & & & 1 & & & & & 3 & & & $\because$ \\
\hline Mangagat & & & & & & & & & & & & & & & 1 & & & & & $\simeq$ & & 1 & & & & & & & & & & & & 1 & & $\because$ \\
\hline Mol-mol & & & & & & & 1 & 1 & & & & 1 & & & & & 1 & & & & $\bar{m}$ & & & & & 1 & & 1 & & 1 & & & & & & $\infty$ \\
\hline Pakol & & & & & & & 1 & & & & & & & & & & & & & & 1 & $\stackrel{\infty}{-}$ & & & & & & & & 1 & & & & & & $\bar{N}$ \\
\hline Palad & & & & & & & 1 & & & & & & & & & & & & & & & & ป & & & & & & & & & & & & & $\pi$ \\
\hline Panit & & & & & & & & & & & & 1 & & & & & & & 1 & & & & & $=$ & & & & & & & & & & & & 9 \\
\hline Pating & 1 & 2 & & & & & & & & & & & & & & & & & & & & & & & $\bar{N}$ & & & & & & & & & & & A \\
\hline Sagisi-on & & & & & 1 & & 1 & & & & & & & & & & & & & & & & & & & $\infty$ & & & & & & & & 1 & & $=$ \\
\hline Sapsap & & & & & & & & & & & 2 & & & & & & & & & & & & & & & & 9 & & & & & & 1 & & & $\underline{0}$ \\
\hline Siri & & & & & & & 2 & & 1 & & & 1 & 1 & & & & & & & & & & & & & & & $=$ & & & & & 1 & & & ת \\
\hline Sulid & & & & & & & & & & & & & & & & \begin{tabular}{|l|}
1 \\
\end{tabular} & & & & 1 & & & & & & & & 1 & $\simeq$ & & & & & & & $\because$ \\
\hline Surahan & & & & & & & 2 & & & & & & & & & \begin{tabular}{|l|}
2 \\
\end{tabular} & & & & & 1 & 1 & & & & & & & & $\tilde{\lambda}$ & 1 & & & & & is \\
\hline Talakitok & & & & & & & & & & & 1 & & & & & & & & & & & & & & & & & 1 & & & $=$ & & & & & 9 \\
\hline Tamban & & & & & & & & & & & & 1 & & & & & & & & & & & & 1 & & & & & & & & $\bar{\lambda}$ & & 1 & & A \\
\hline Tarukitok & & & & & & & & & & & & & & & & & & & & & & & & & & & & & & & 1 & & 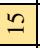 & & & $\underline{6}$ \\
\hline Ti-aw & & & & & & & & 1 & & & & & 1 & & & & & & & & & & & & & & & & 1 & & & 1 & & 2 & 1 & A \\
\hline Tingag & & & & 1 & & & 3 & & & & & 1 & & & & 3 & & & & & & & & & & & & & & 1 & & & & & m & $F$ \\
\hline Predicted total & \pm & $\stackrel{\infty}{-}$ & $=$ & $=$ & $a$ & ป & in & 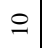 & 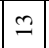 & $=$ & $=$ & iे & \pm & 9 & ส & 强 &  & $a$ & $\simeq$ & 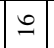 & $m$ & $\dot{A}$ & $\pi$ & $\simeq$ & ส & 오 & 9 & 2 & 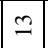 & iे & 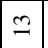 & A & $\bar{N}$ & $\stackrel{\sim}{\sim}$ & F & है \\
\hline
\end{tabular}

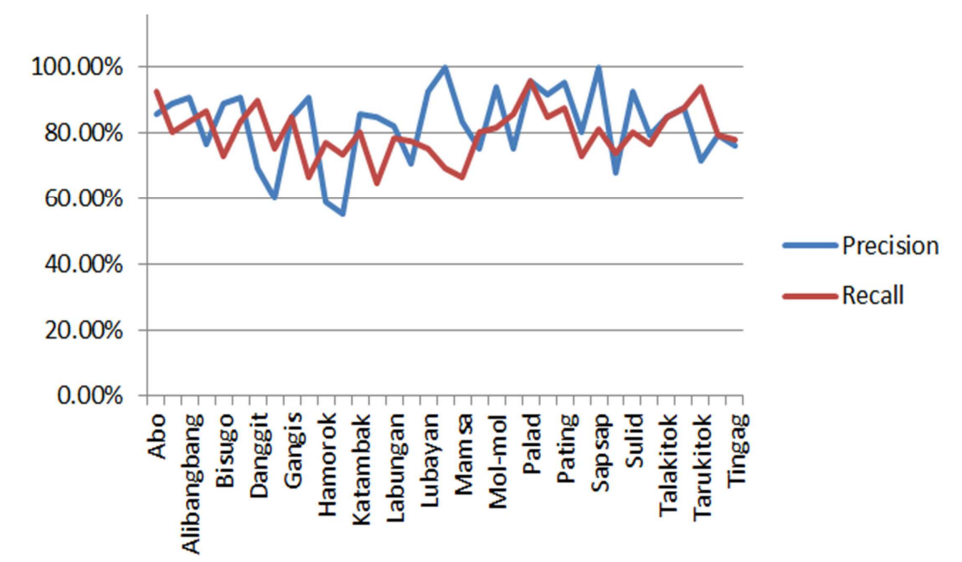

Fig. 10 Precision versus recall

Table 3 presents the results of calculating some of the performance measures that can be derived from the confusion matrix. Palad, as observed also in Fig. 10, earns the highest recall of 95.65\%, and Hangit suffers from low precision of 55\%, which denotes that Hangit positive values are unpredictable. However, only a few of these positive predictions are correct. On 
the other hand, Malatindok and Sapsap have the highest precision of $100 \%$ while Kirawan garnered the lowest recall of $64.71 \%$. This is a manifestation that the model is returning very few results, yet most of its predicted labels are correct when compared to the training labels. Moreover, Malatindok and Sapsap classes obtain the highest specificity percentage which signifies that the model is $100 \%$ accurate in recognizing the fish images.

Table 3 Performance evaluation result

\begin{tabular}{|c|c|c|c|}
\hline Class & Precision & Recall & Specificity \\
\hline Abo & $85.71 \%$ & $92.31 \%$ & $99.70 \%$ \\
\hline Alho & $88.89 \%$ & $80.00 \%$ & $99.70 \%$ \\
\hline Alibangbang & $90.91 \%$ & $83.33 \%$ & $99.85 \%$ \\
\hline Baga-baga & $76.47 \%$ & $86.67 \%$ & $99.40 \%$ \\
\hline Bisugo & $88.89 \%$ & $72.73 \%$ & $99.85 \%$ \\
\hline Bon-ak & $90.91 \%$ & $83.33 \%$ & $99.70 \%$ \\
\hline Danggit & $69.23 \%$ & $90.00 \%$ & $97.52 \%$ \\
\hline Dumpilas & $60.00 \%$ & $75.00 \%$ & $99.41 \%$ \\
\hline Gangis & $84.62 \%$ & $84.62 \%$ & $99.70 \%$ \\
\hline Ganting & $90.91 \%$ & $66.67 \%$ & $99.85 \%$ \\
\hline Hamorok & $58.82 \%$ & $76.92 \%$ & $98.96 \%$ \\
\hline Hangit & $55.00 \%$ & $73.33 \%$ & $98.66 \%$ \\
\hline Katambak & $85.71 \%$ & $80.00 \%$ & $99.70 \%$ \\
\hline Kirawan & $84.62 \%$ & $64.71 \%$ & $99.70 \%$ \\
\hline Labungan & $81.82 \%$ & $78.26 \%$ & $99.40 \%$ \\
\hline Lapu-lapu & $70.45 \%$ & $77.50 \%$ & $97.98 \%$ \\
\hline Lubayan & $92.31 \%$ & $75.00 \%$ & $99.85 \%$ \\
\hline Malatindok & $100.00 \%$ & $69.23 \%$ & $100.00 \%$ \\
\hline Mamsa & $83.33 \%$ & $66.67 \%$ & $99.70 \%$ \\
\hline Mangagat & $75.00 \%$ & $80.00 \%$ & $99.40 \%$ \\
\hline Mol-mol & $93.94 \%$ & $81.58 \%$ & $99.69 \%$ \\
\hline Pakol & $75.00 \%$ & $85.71 \%$ & $99.09 \%$ \\
\hline Palad & $95.65 \%$ & $95.65 \%$ & $99.85 \%$ \\
\hline Panit & $91.67 \%$ & $84.62 \%$ & $99.85 \%$ \\
\hline Pating & $95.45 \%$ & $87.50 \%$ & $99.85 \%$ \\
\hline Sagisi-on & $80.00 \%$ & $72.73 \%$ & $99.70 \%$ \\
\hline Sapsap & $100.00 \%$ & $81.25 \%$ & $100.00 \%$ \\
\hline Siri & $68.00 \%$ & $73.91 \%$ & $98.79 \%$ \\
\hline Sulid & $92.31 \%$ & $80.00 \%$ & $99.85 \%$ \\
\hline Surahan & $79.31 \%$ & $76.67 \%$ & $99.08 \%$ \\
\hline Talakitok & $84.62 \%$ & $84.62 \%$ & $99.70 \%$ \\
\hline Tamban & $87.50 \%$ & $87.50 \%$ & $99.55 \%$ \\
\hline Tarukitok & $71.43 \%$ & $93.75 \%$ & $99.10 \%$ \\
\hline Ti-aw & $79.17 \%$ & $79.17 \%$ & $99.24 \%$ \\
\hline Tingag & $76.19 \%$ & $78.05 \%$ & $98.45 \%$ \\
\hline
\end{tabular}

\subsection{Graphical user interface}

The graphical user interface for the mobile-based application is created using Android Studio 4.1 in which the model is embedded in the application. The generated model, which is a Keras file (.h5), is converted into a Tensorflow file (.tflite) using TensorFlow Lite which is a set of tools to help developers run TensorFlow models on mobile. This file is then deployed into the mobile-based application. The graphical user interface of the application is shown in Figs. 11 (a) and (b) which display the final prediction of the classification model on the uploaded or selected image as well as the necessary information of the predicted fish. 


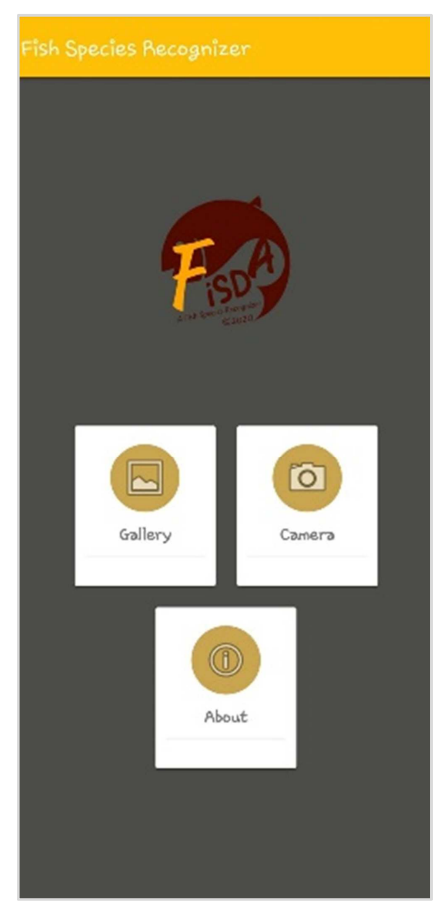

(a) Main interface of the application

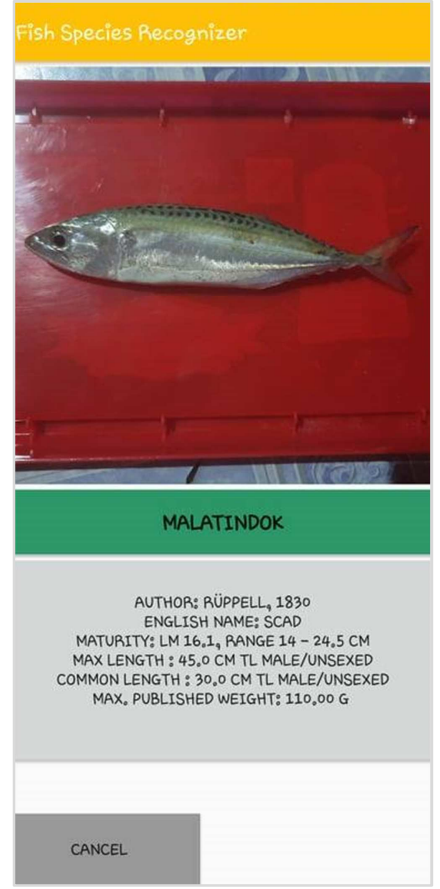

(b) Recognition result interface

Fig. 11 Graphical user interface of the application

\section{Conclusions and Recommendations}

This study was able to come up with a fish species recognizer model which was successfully embedded in a mobile-based application. By using CNN, the generated model obtained a training accuracy rate of $96.49 \%$ somehow higher than the study of Pudaruth et al. [19], which only gained 96\% using k-nearest neighbors (kNN) classifier. During the validation process, the model achieved $82.81 \%$ accuracy with a loss value of 1.868 , which indicates that the model has good reliability when it comes to predicting the fish images. Moreover, during the testing process, the model gained an accuracy rate of $80.58 \%$. The result revealed that the model performs well in predicting Malatindok and Sapsap species, which gained the highest precision of $100 \%$. It is because of their distinct morphological features such as shape and color. On the other hand, Hangit was sometimes misclassified by the model after obtaining a 55\% accuracy rate from the testing results because of insufficient dataset for this specific species as well as of its feature similarity to other species. In the future, there is a need to further improve the performance of the model by using more datasets of fish images to have a better prediction result.

\section{Conflicts of Interest}

The authors declare no conflict of interest.

\section{References}

[1] J. Davies, P. M. Magsalay, R. Rigor, A. Mapalo, and H. Gonzales, "Directory of Philippine Wetlands: A Preliminary Compilation of Information on Wetlands of the Philippines," Philippines: Asian Wetland Bureau Philippines Foundation, 1990.

[2] M. C. Francisco, N. A. Dayap, L. A. Tumabiene, R. A. Francisco, M. J. Candole, J. H. De Veyra, et al., "Status of Leyte Gulf Fisheries CYs 2001-2011," The Philippine Journal of Fisheries, vol. 25, no. 1, pp. 136-155, 2018.

[3] K. F. Lagler, J. E. Bardach, and R. R. Miller, "Ichthyology: The Study of Fishes,” New York: John Wiley \& Sons, 1962.

[4] D. Rathi, S. Jain, and S. Indu, "Underwater Fish Species Classification Using Convolutional Neural Network and Deep Learning," 9th International Conference on Advances in Pattern Recognition, December 2017, pp. 1-6.

[5] J. Jäger, E. Rodner, J. Denzler, V. Wolff, and K. Fricke-Neuderth, "SeaCLEF 2016: Object Proposal Classification for Fish Detection in Underwater Videos,” 7th Conference and Labs of the Evaluation Forum, September 2016, pp. 481-489. 
[6] X. Lan, J. Bai, M. Li, and J. Li, "Fish Image Classification Using Deep Convolutional Neural Network,” International Conference on Computers, Information Processing, and Advanced Education, October 2020, pp. 18-22.

[7] F. Shafait, A. Mian, M. Shortis, B. Ghanem, P. F. Culverhouse, D. Edgington, et al., "Fish Identification from Videos Captured in Uncontrolled Underwater Environments," ICES Journal of Marine Science, vol. 73, no. 10, pp. 2737-2746, November 2016.

[8] J. Fischer, Fish Identification Tools for Biodiversity and Fisheries Assessments: Review and Guidance for Decision-Makers, Rome: Food and Agriculture Organization of the United Nations, 2013.

[9] M. M. M. Fouad, H. M. Zawbaa, N. El-Bendary, and A. E. Hassanien, “Automatic Nile Tilapia Fish Classification Approach Using Machine Learning Techniques," 13th International Conference on Hybrid Intelligent Systems, December 2013, pp. 173-178.

[10] A. Domínguez, “A History of the Convolution Operation,” IEEE Pulse, vol. 6, no. 1, pp. 38-49, January-February 2015.

[11] M. A. Iqbal, Z. Wang, Z. A. Ali, and S. Riaz, "Automatic Fish Species Classification Using Deep Convolutional Neural Networks," Wireless Personal Communications, vol. 116, no. 2, pp. 1043-1053, January 2021.

[12] N. E. M. Khalifa, M. H. N. Taha, and A. E. Hassanien, “Aquarium Family Fish Species Identification System Using Deep Neural Networks," International Conference on Advanced Intelligent Systems and Informatics, September 2018, pp. 347-356.

[13] J. H. Christensen, L. V. Mogensen, R. Galeazzi, and J. C. Andersen, "Detection, Localization and Classification of Fish and Fish Species in Poor Conditions using Convolutional Neural Networks," IEEE/OES Autonomous Underwater Vehicle Workshop, November 2018, pp. 1-6.

[14] B. S. Rekha, G. N. Srinivasan, S. K. Reddy, D. Kakwani, and N. Bhattad, "Fish Detection and Classification Using Convolutional Neural Networks," International Conference on Computational Vision and Bio-Inspired Computing, September 2019, pp. 1221-1231.

[15] J. N. Fabic, I. E. Turla, J. A. Capacillo, L. T. David, and P. C. Naval, "Fish Population Estimation and Species Classification from Underwater Video Sequences Using Blob Counting and Shape Analysis,” IEEE International Underwater Technology Symposium, March 2013, pp. 1-6.

[16] K. M. Knausgård, A. Wiklund, T. K. Sørdalen, K. T. Halvorsen, A. R. Kleiven, L. Jiao, et al., "Temperate Fish Detection and Classification: A Deep Learning Based Approach," Applied Intelligence, in press.

[17] L. Wang, "WeFish: An Interactive Information Design Mobile App for Local Fishing," Thesis, College of Imaging Arts and Sciences, Rochester Institute of Technology, New York, NY, 2017.

[18] R. Froese and D. Pauly, “2021 FishBase,” http://www.fishbase.org, June 2021.

[19] S. Pudaruth, N. Nazurally, C. Appadoo, S. Kishnah, M. Vinayaganidhi, I. Mohammoodally, et al., "SuperFish: A Mobile Application for Fish Species Recognition Using Image Processing Techniques and Deep Learning," International Journal of Computing and Digital Systems, vol. 10, no. 1, pp. 1-14, January 2021.

Copyright $(\mathrm{C}$ by the authors. Licensee TAETI, Taiwan. This article is an open access article distributed under the terms and conditions of the Creative Commons Attribution (CC BY-NC) license (https://creativecommons.org/licenses/by-nc/4.0/). 